\title{
Formation of Polyrotaxane Particles via Template
}

\section{Assembly}

Blaise L. Tardy, ${ }^{a, \ddagger}$ Shereen Tan, ${ }^{b, \ddagger}$ Henk H. Dam, ${ }^{a}$ Tomoya Suma, ${ }^{a}$ Junling Guo, ${ }^{a}$ Greg G. Qiao, $^{b}$ and Frank Caruso ${ }^{a, *}$

${ }^{a}$ ARC Centre of Excellence in Convergent Bio-Nano Science and Technology, and the Department of Chemical and Biomolecular Engineering, the University of Melbourne, Parkville, Victoria 3010, Australia

${ }^{\mathrm{b}}$ Polymer Science Group, Department of Chemical and Biomolecular Engineering, the University of Melbourne, Parkville, Victoria 3010, Australia.

\section{KEYWORDS}

polyrotaxane particles, polyrotaxane capsules, cyclodextrin, template assembly, host guest chemistry 


\section{ABSTRACT}

In this study, we report a versatile method to assemble tunable poly(ethylene glycol) (PEG)based polyrotaxane (PRX) particles and capsules. By threading $\alpha$-cyclodextrins ( $\alpha \mathrm{CDs})$ onto PEG chains physically adsorbed onto template particles and subsequently dissolving the templates, PRX replica particles and hollow capsules are formed. This approach overcomes issues related to CD steric hindrance, and also reduces the multiple processing steps often associated with PRX-based particle formation. By simple variation of the molecular weight and end-group functionality of the PEG, we show that the rate of particle degradation rate as well as the stability of the particles can be tuned. We also demonstrate the loading and release of model (drug) compounds, achieving burst and controlled release of the compounds. It is envisaged that this approach will provide a flexible platform for the engineering of a diverse range of PRX-based particles, enabling PRX materials to be further explored in various applications. 


\section{INTRODUCTION}

Polymer-based particle carriers have received widespread interest due to their ability to store and selectively release cargo on demand, ${ }^{1,2}$ and have been explored for a range of applications, including targeted drug delivery, ${ }^{3,4}$ gene therapy ${ }^{5}$ microreactions, ${ }^{6}$ sensing, ${ }^{7,8}$ and catalysis. ${ }^{2,3,9-12}$ Significant effort has been directed toward the design and improvement of the assembly protocols, ${ }^{8,13}$ often with a focus on controlling particle morphology, size, composition, and functionality. Commonly used methods include template-assisted ${ }^{13-17}$ and self-assembly ${ }^{18-20}$ approaches. The template-assisted approach utilizes a sacrificial template where polymers can be deposited onto the surface of the template and cross-linked or modified in situ or post-deposition. Upon template dissolution, cross-linked particles or hollow capsules are obtained. Templating methods allow for a high degree of control over the properties of the particles, including size, architecture and polydispersity, and also lend themselves to simple isolation and purification procedures. ${ }^{13}$

Coating approaches that are used in conjunction with template-assisted assembly include layer-by-layer (LbL) assembly, $^{8}$ (bio-inspired) self-assembly ${ }^{21}$ and interfacial polymerization. ${ }^{15,22,23}$ The LbL approach offers nanometer resolution over the thickness of the materials deposited, a wide range of materials selection, and a number of processing alternatives, including immersion, spray, microfluidics, electrophoretic and fluidized bed technologies, with some of these amenable to automation, which is particularly important for industrial applications. ${ }^{24}$ LbL assembly is a cyclical process, where conventionally, a charged material is firstly adsorbed onto a substrate, and after washing steps, a complementary charged material is adsorbed on top of the first layer. This typically results in a single bilayer with nanometer-scale thickness of the adsorbed bilayer. This deposition process can also be repeated until multilayer films of a desired thickness are achieved. Although electrostatic interactions remain widely used to facilitate film buildup, ${ }^{25}$ other molecular interactions 
including covalent, ${ }^{26}$ hydrogen bonding ${ }^{27-29}$ and supramolecular chemistry ${ }^{23}, 30$ have been explored. Specifically, polymeric supramolecular chemistry is a relatively new area of significant interest as the interactions are highly specific, yet reversible. ${ }^{31}$

Supramolecular chemistry based on inclusion chemistry involves a range of noncovalent interactions between a 'host' (e.g. cyclodextrin) and a 'guest' to form higher ordered reversible constructs via a self-assembly process. ${ }^{32,33}$ Cyclodextrins (CDs) are one of the most commonly used hosts due to their wide availability and low toxicity. Owing to their hydrophobic interior cavity, CDs are able to form reversible interactions with appropriately sized guest molecules and polymers. ${ }^{32}, 33 \alpha \mathrm{CD}$ complexation with poly(ethylene glycol) (PEG) and its subsequent end-capping to permanently trap the CDs onto the PEG chains forms complexes termed polyrotaxanes (PRXs). Such constructs were first discovered by Harada and co-workers ${ }^{34-36}$ and exhibit interesting properties that cannot be easily achieved using covalent analogues. Some of these novel features include: (i) rotational and translational freedom about the main chain without disassociation; (ii) high tenability, where the rigidity of the complex can be controlled by threading degree; and (iii) increased multivalent interactions where the threaded CDs can be functionalized with ligands. ${ }^{37,38}$ Owing to these attributes, PEG-based PRXs have been extensively studied for macroscale applications. $^{33,39,40}$ The use of PRX-based materials in the form of free floating, nanoscale, thin films remains largely unexplored, with only several reports mainly utilizing LbL assembly for film formation. ${ }^{41-43}$ For example, we recently reported the fabrication of degradable PRX multilayer films and capsules formed using electrostatic interactions. ${ }^{42,43}$ The threaded CDs were modified with complementary charged groups to provide noncovalent cross-linking sites for the iterative deposition of the two PRXs. ${ }^{46,47}$ The formation of stabilized capsules required specific conditions, such as a high threading degree, eight layering steps, and the pre-synthesis of PRXs. ${ }^{43}$ These stringent conditions are attributed to 
the rigidity of the rod-like PRXs, which makes it challenging to form a sufficient number of intra-and inter-molecular cross-linking sites, which is essential for film stability.

With a view to overcoming these limitations, herein we report a simple and tunable approach for the formation of template-assisted CD-based PRX particles and capsules. To the best of our knowledge, this is the first report of a one-pot, highly tunable method toward the formation of hollow capsules and replica particles. In this approach, non-porous and mesoporous silica $\left(\mathrm{SiO}_{2}\right)$ templates are pre-coated with PEG. The adsorbed PEG on the surface is essential, as sufficient bonding between the $\mathrm{SiO}_{2}$ surface and PEG is required for stability, while free PEG chain ends known as 'tails' are the sites for inclusion complexation with $\alpha \mathrm{CD}$ (Scheme 1, step a). The formed constructs are subsequently suspended in a concentrated solution of $\alpha \mathrm{CD}$, which allows for the directed assembly of CDs onto the PEG chains, thus forming pseudopolyrotaxanes (PPRXs). Over time, the threaded CDs hydrogen bond with each other to create crystalline domains that act as cross-linking sites (Scheme 1, step b). Upon template removal, discrete hollow capsules and replica particles are obtained (Scheme 1, step c). By changing the $\alpha \mathrm{CD}$ concentration, the rate of particle disassembly can be tuned. Furthermore, varying the molecular weight and end-groups of the PEG backbone also affects the size, degradation rate, and particle swellability. Model drug release studies from the particles reveal that the constructs can be further tuned to replicate burst and controlled release profiles. Covalent particle stabilization was achieved by forming bridges between the end groups of threaded multi-armed PEGs. Due to the simple, yet versatile nature of this approach, it is anticipated that new avenues for PPRX and PRX-based materials will emerge, especially at the micron-scale, where steric hindrance (i.e., bulky nature of the CD molecule hindering accessibility to small molecules) commonly plays a significant inhibitory role. 

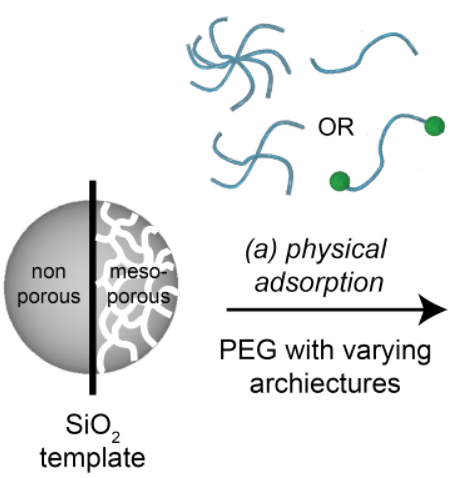

(a) physical adsorption archiectures template

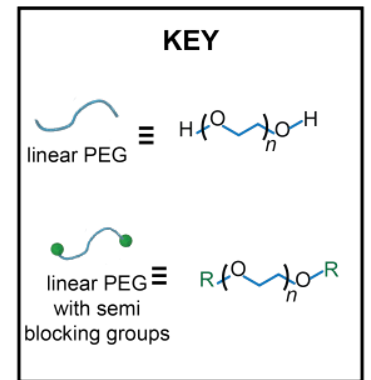

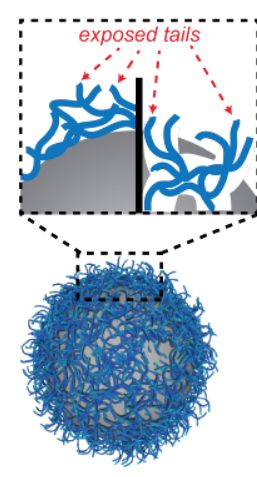

physically adsorbed PEG
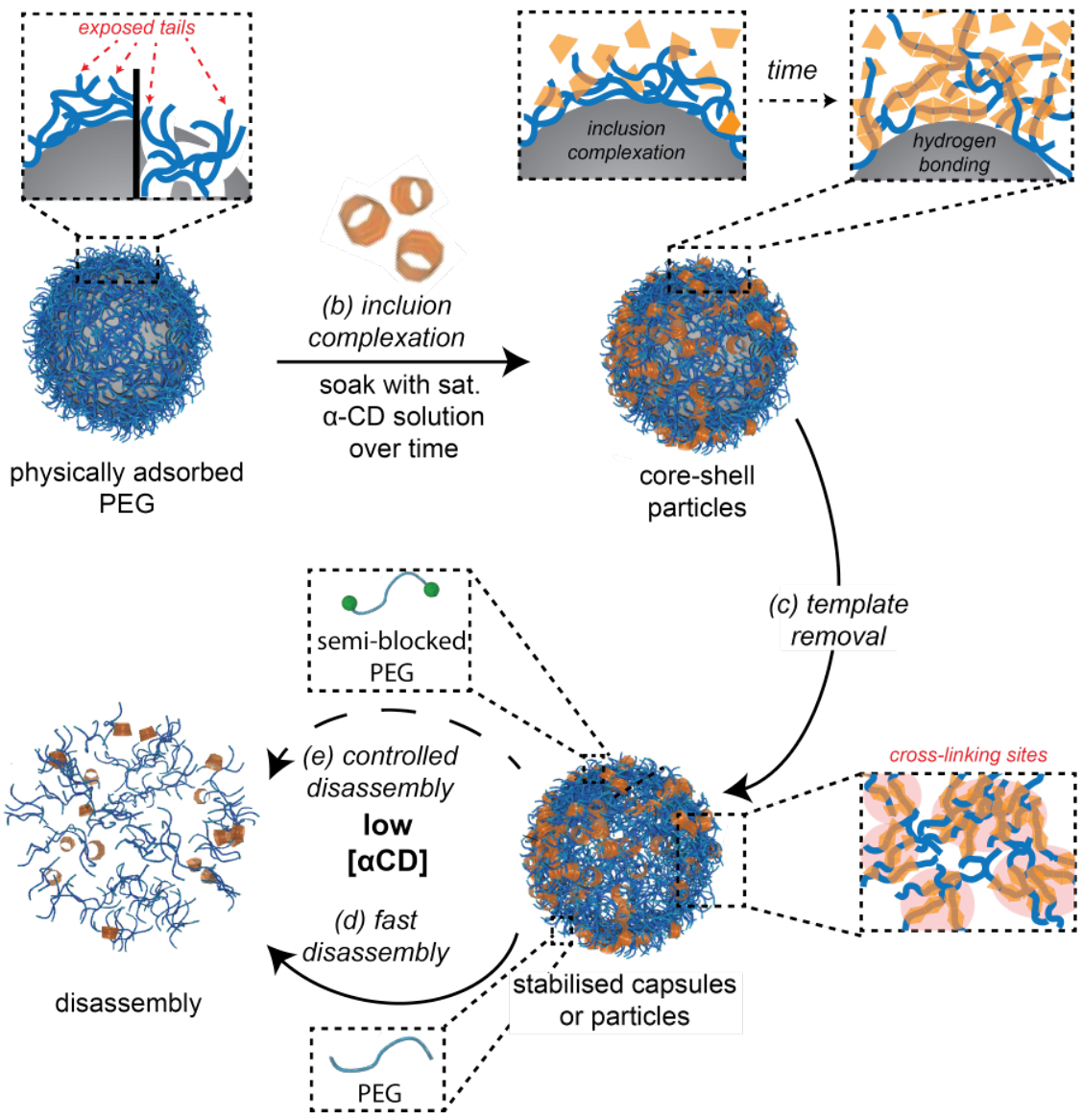

Scheme 1. Formation of PPRX particles and capsules by firstly (a) adsorbing PEG of different molecular weights or with different end-groups on a $\mathrm{SiO}_{2}$ particle. This is followed by (b) suspending the formed construct in a saturated solution of $\alpha \mathrm{CD}$. This directs the threading of $\alpha \mathrm{CDs}$ onto the adsorbed PEG through the PEG tails. Over time, the threaded $\alpha \mathrm{CDs}$ hydrogen bond both inter- and intramolecularly. (c) After core dissolution, stable replica particles and hollow capsules, stabilized by CD rich crystalline domains are formed. (d) Upon lowering the surrounding [ $\alpha \mathrm{CD}]$, particle disassembly can be achieved. (e) The rate of disassembly can also be controlled by using PEGs with various semi-blocking groups.

\section{EXPERIMENTAL SECTION}

Materials. High-purity Milli-Q (MQ) water with a resistivity greater than $18 \mathrm{M} \Omega \cdot \mathrm{cm}$ was obtained from an in-line Millipore RiOs/Origin water purification system. The $\mathrm{pH}$ of solutions was measured with a Mettler-Toledo MP220 pH meter. $\alpha \mathrm{CD}$ was obtained from 
Kanto Chemical Co. (Japan). Doxorubicin hydrochloride, PEG ( $M_{w}:$ 1.1, 3.35, 6, 10, 20 and $100 \mathrm{~g} \mathrm{~mol}^{-1}$ ), 2-methoxyphenol, 2,5-dimethylphenol, 2-ethylphenol, 2-propylphenol, 2isopropylphenol, phosphate buffered saline (PBS) tablets, sodium acetate, rhodamine B octadecyl ester perchlorate (RhD-C), p-toluene sulfonyl chloride (TsCl), dimethyl sulfoxide (DMSO), dichloromethane (DCM), acetone, diethyl ether (DEE), deuterium oxide, deuterated DMSO (DMSO- $\left.d_{6}\right)$, deuterated chloroform $\left(\mathrm{CDCl}_{3}\right)$, glutaraldehyde, sodium borohydride, hydrofluoric acid (HF), ammonium fluoride $\left(\mathrm{NH}_{4} \mathrm{~F}\right)$, and Triton- $\mathrm{X}$ were obtained from Sigma-Aldrich (Australia). Amine-terminated 4-arm and 8-arm PEG ( $M_{w} 40$ and $10 \mathrm{~g} \mathrm{~mol}^{-1}$, respectively) were obtained from JenKem Technology, USA. All chemicals were used as received. Silica particles were obtained from Microparticles GmbH (Germany). Mesoporous silica particles (MPSP), and Separon SGX 200 and 500, were purchased from Tessek Ltd (Czech Republic).

Replica Particle Formation. $2 \mathrm{mg} \mathrm{mL}^{-1}$ MSPSs (average diameter $5 \mu \mathrm{m}$, pore size $\sim 50$ $\mathrm{nm}$ ) in MQ water was mixed with a solution of $2 \mathrm{mg} \mathrm{mL}^{-1} \mathrm{PEG}$ in MQ water at a 1:1 volume ratio. The suspension was sonicated for 15 min then shaken for $12 \mathrm{~h}$ on a rotary tube mixer. The particles were isolated by centrifugation (500-3000 $\mathrm{g}$, $1 \mathrm{~min}$ ), the supernatant was then replaced by a solution of $100 \mathrm{mg} \mathrm{mL}^{-1}$ of $\alpha \mathrm{CD}$ in MQ water. After $12 \mathrm{~h}$ shaking on a rotary tube mixer, the suspension was mixed with $5 \mathrm{M} \mathrm{HF}$ in $50 \mathrm{mg} \mathrm{mL}^{-1} \alpha \mathrm{CD}$ at a 1:1 volume ratio to remove the $\mathrm{SiO}_{2}$ template. The sample was then washed three times with MQ water in the presence of $50 \mathrm{mg} \mathrm{mL}^{-1} \alpha \mathrm{CD}$.

Capsule Formation. $5 \mathrm{mg} \mathrm{mL}^{-1} \mathrm{SiO}_{2}$ particles (average diameter $5 \mu \mathrm{m}$ ) was mixed at a 1:1 volume ratio with a solution of $2 \mathrm{mg} \mathrm{mL}^{-1} \mathrm{PEG}$ in MQ water. The suspension was sonicated for $15 \mathrm{~min}$ then shaken for $12 \mathrm{~h}$ on a rotary tube mixer. The particles were isolated by centrifugation (3000 $\mathrm{g}, 1 \mathrm{~min}$ ) and the supernatant was then replaced by a solution of $100 \mathrm{mg}$ $\mathrm{mL}^{-1}$ of $\alpha \mathrm{CD}$ in MQ water. After shaking the suspension for $7 \mathrm{~d}$ on a rotary tube mixer, the 
suspension was mixed at a 1:1 volume ratio with a solution of $5 \mathrm{M} \mathrm{HF}$ in $50 \mathrm{mg} \mathrm{mL}^{-1} \alpha \mathrm{CD}$ to remove the $\mathrm{SiO}_{2}$ template. The sample was then washed three times with MQ water in the presence of $50 \mathrm{mg} \mathrm{mL}^{-1} \alpha \mathrm{CD}$.

aCD Concentration Dependent Degradation Assay. A suspension of replica particles (RPs) formed using MSPSs (average diameter $5 \mu \mathrm{m}$, $50 \mathrm{~nm}$ pore size) with bis- $\mathrm{OH}_{2}$ terminated PEG (Mw $3.35 \mathrm{kDa}$ ), was used for the concentration dependent degradation assay. $\mathrm{UV}$-Vis spectroscopy was used to measure the degradation rates of the RPs. Degradation was achieved by diluting the suspension with PBS containing varying amounts of $\alpha \mathrm{CD}$ in order to obtain the desired $\alpha \mathrm{CD}$ concentration and an initial $\left(\mathrm{t}_{0}\right)$ absorbance value of $\sim 0.25$. During the experiment, the samples were shaken at $1600 \mathrm{rpm}$ on a thermomixer at $37^{\circ} \mathrm{C}$. A series of 3 samples were prepared for each concentration and measured using a NanoDrop 3300 instrument at $500 \mathrm{~nm}$ at given time points. Data points plotted are the average of three individual experiments.

Semi-Blocked PEG RP Formation and Degradation. $2 \mathrm{mg} \mathrm{mL}^{-1} \mathrm{MSPSs}$ (diameter $5 \mu \mathrm{m}$, pore size $\sim 50 \mathrm{~nm}$ ) in MQ water was mixed with a solution of $2 \mathrm{mg} \mathrm{mL}^{-1}$ PEG functionalized with semi blocking groups in MQ water at a 1:1 volume ratio. The suspension was then sonicated for $15 \mathrm{~min}$ and shaken on a rotary tube mixer for $12 \mathrm{~h}$. The particles were isolated by centrifugation (700 $\mathrm{g}, 1 \mathrm{~min}$ ) and the supernatant was replaced by a solution of $140 \mathrm{mg}$ $\mathrm{mL}^{-1} \alpha \mathrm{CD}$ in MQ water ( $[\alpha C D]=210 \mathrm{mg} \mathrm{mL}^{-1}$ when $\mathrm{R}_{4}$ was used). After shaking the suspension on a rotary tube mixer for more than 2 weeks, the suspension was mixed at a 1:1 volume ratio with a solution of $5 \mathrm{M} \mathrm{HF}$ to remove the $\mathrm{SiO}_{2}$ template. The sample was then washed three times with PBS in the presence of $50 \mathrm{mg} \mathrm{mL} L^{-1} \alpha C D$. Degradation was monitored using a Nano ZS model ZEN3600 instrument. From stock solutions containing a concentrated suspension of RPs, a suspension was prepared by dilution with a solution of 50 $\mathrm{mg} \mathrm{mL} \mathrm{m}^{-1} \alpha \mathrm{CD}$ in PBS in order to obtain a scattering signal of ca. 25000 kilo counts per 
seconds (kCPS, taken as $\mathrm{t}_{0}$ ). The same quantity used for this dilution was then added to PBS containing varying amount of $\alpha \mathrm{CD}$ s in order to obtain a maximum, fixed, final concentration of $\alpha \mathrm{CD}$ of $2.5 \mathrm{mg} \mathrm{mL}^{-1}$. The scattering intensity was then averaged and reported every $20 \mathrm{~s}$. Every 10 min, the sample was mixed by pipetting to avoid sedimentation. A moving average over 5 points was applied to limit overlap between the curves for the data reported using semi-blocking groups $\mathrm{R}_{3}, \mathrm{R}_{4}$, and $\mathrm{R}_{5}$.

Cargo loading and release from Replica Particles. MSPSs $(3 \mathrm{mg}$, diameter $=5 \mu \mathrm{m}$, pore size $\sim 20 \mathrm{~nm})$ was suspended $0.4 \mathrm{~mL}$ of an aqueous solution of PEG $\mathrm{R}_{1}\left(1 \mathrm{mg} \mathrm{mL}^{-1}, 3.35\right.$ $\mathrm{kDa}$ ). The suspension was sonicated for $15 \mathrm{~min}$ and subsequently agitated for $12 \mathrm{~h}$ on a rotary tube mixer. PPRX formation and drug loading when then conducted. The particles was firstly isolated by centrifugation (3000 g, $1 \mathrm{~min}$ ) and the supernatant was replaced with, (i) $0.4 \mathrm{~mL}$ of a saturated aqueous solution of $\alpha \mathrm{CD}\left(100 \mathrm{mg} \mathrm{mL}^{-1}\right)$, and (ii) $1.6 \mu \mathrm{L}$ of a solution of doxorubicin (DOX, $100 \mathrm{mg} \mathrm{mL}^{-1}$ in DMSO), or a solution of Rhodamine-C (RhD-C, $10 \mathrm{mg}$ $\mathrm{mL}^{-1}$ in DMSO). For the release experiments, the suspension was shaken for a further (2 days (DOX) and 2 weeks (RhD-C), respectively) on a rotary tube mixer and core dissolution was achieved by mixing the suspension in a 1:1 volume ratio with a solution containing $5 \mathrm{M} \mathrm{HF}$ and 13.3 $\mathrm{M} \mathrm{NH}_{4} \mathrm{~F}$ at a 1:2 volume ratio. The suspension was subsequently washed 9 times (centrifuge condition: $700 \mathrm{~g}, 1 \mathrm{~min}$ ) with $\mathrm{PBS}$ in the presence of $\alpha \mathrm{CD}\left(50 \mathrm{mg} \mathrm{mL}{ }^{-1}\right)$. For DOX cargo release tests, $25 \mu \mathrm{L}$ the DOX loaded replica particle suspension was diluted with $1.475 \mathrm{ml}$ of PBS and shaken at $1600 \mathrm{rpm}$ in a thermomixer at $37{ }^{\circ} \mathrm{C}$. At various time points, the solution was centrifuged (3000 g, $2 \mathrm{~min}$ ) and $100 \mu \mathrm{L}$ of the supernatant was mixed with $100 \mu \mathrm{L}$ of a solution of $0.1 \mathrm{M}$ sodium acetate at $\mathrm{pH} 4$ (to avoid self- quenching of doxorubicin). For Rhd-C cargo release tests, $45 \mu \mathrm{L}$ the DOX loaded replica particle suspension was diluted with $1.855 \mathrm{ml}$ of PBS and shaken at $1600 \mathrm{rpm}$ in a thermomixer at 37 ${ }^{\circ} \mathrm{C}$. At various time points, the solution was centrifuged $(1700 \mathrm{~g}, 2 \mathrm{~min})$ and $100 \mu \mathrm{L}$ of the 
supernatant was mixed with $50 \mu \mathrm{L}$ of $0.1 \mathrm{M}$ sodium acetate and $50 \mu \mathrm{L}$ of Triton-X, to avoid quenching of the alkyl modified RhD-C by micellization. The data points plotted are the average of three individual experiments. Normalized values were obtained by comparing to $\mathrm{T}_{0}$ values. $\mathrm{T}_{0}$ value was achieved by mixing $100 \mu \mathrm{L}$ of the original suspension (prior to centrifugation) with $100 \mu \mathrm{L}$ of sodium acetate $(0.1 \mathrm{M})$ (and $50 \mu \mathrm{L}$ of Triton-X for Rhd-C release) followed by sonication for 2 min.

Formation of Cross-Linked Particles from Amine Terminated 4- and 8-Arm PEGs. The RPs containing multi-armed PEGs were formed in a similar way as for the RPs described above, but prior to template removal a cross-linking step was added. Amine terminated 8-arm PEGs $\left(500 \mu \mathrm{L}, M_{w} 10 \mathrm{~kg} \mathrm{~mol}^{-1}, 2 \mathrm{mg} \mathrm{mL}^{-1}\right)$ or 4-arm PEGs $\left(500 \mu \mathrm{L}, M_{w} 40 \mathrm{~kg} \mathrm{~mol}^{-1}, 2 \mathrm{mg}\right.$ $\mathrm{mL}^{-1}$ ) were adsorbed for $12 \mathrm{~h}$ on MSPSs $(1 \mathrm{mg}$, diameter $5 \mu \mathrm{m}$, pore size $\sim 50 \mathrm{~nm})$. The particles were isolated by centrifugation (3000 $\mathrm{g}, 1 \mathrm{~min}$ ) and then the supernatant was replaced by $2 \mathrm{~mL}$ of $100 \mathrm{mg} \mathrm{mL}^{-1}$ of $\alpha \mathrm{CD}$ in MQ water. After $12 \mathrm{~h}$, glutaraldehyde was added to a final concentration of $0.2 \mathrm{mg} \mathrm{mL}^{-1}$ for $8 \mathrm{~h}$ in phosphoric acid solution (pH 8, 50 $\mathrm{mM}$ ) with final concentrations of $\alpha \mathrm{CD}$ of 50 and $12.5 \mathrm{mg} \mathrm{mL}^{-1}$ respectively. The particles were isolated by centrifugation and the supernatant replaced with a solution of sodium borohydride (2 mg) in MQ water $(2 \mathrm{~mL})$. After $6 \mathrm{~h}$, the particles were washed three times with MQ water (centrifuge condition: $3000 \mathrm{~g}, 1 \mathrm{~min}$ ) and the template was removed with HF as previously described. Reversible swelling and shrinkage was monitored by dropping a solution containing the RPs on a slide followed by sequential mixing of the droplet with solutions of $1 \mathrm{M} \mathrm{NaOH}$ or $2 \mathrm{M} \mathrm{HCl}$.

UV-Vis Monitoring of the Formation and Degradation of PPRX from Semi-Blocked PEGs and $\alpha$ CD. The PPRX were formed by mixing $190 \mu \mathrm{L}$ of a solution of $110 \mathrm{mg} \mathrm{mL}^{-1}$ $\alpha \mathrm{CD}$ with $10 \mu \mathrm{L}$ of a solution of $200 \mathrm{mg} \mathrm{mL}^{-1}$ PEG. The absorbance was recorded at $500 \mathrm{~nm}$ on a Cary 50 microplate reader (Varian, CA). For degradation, the resulting PPRX solutions 
were decanted, freeze-dried, and $7 \mathrm{mg}$ of PPRX powder was redissolved in $0.2 \mathrm{~mL}$ of MQ water. The absorbance was measured at $500 \mathrm{~nm}$.

Nuclear Magnetic Resonance (NMR). NMR spectra were recorded using a $400 \mathrm{MHz}$ Varian INOVA system at $25{ }^{\circ} \mathrm{C}$. Spectra were referenced to residual proton resonances of the deuterated solvent. Proton chemical shift values $\left(\delta_{\mathrm{H}}\right)$ are reported as parts per million (ppm) downfield from the signal originating from TMS (tetramethylsilane), referenced to residual protons in the deuterated solvents.

Powder X-ray Diffraction. $100 \mathrm{mg}$ of MSPSs (diameter $5 \mu \mathrm{m}$, pore size $\sim 50 \mathrm{~nm}$ ) were used as templates and the resulting particles (post template removal) were obtained by using a spin column with a membrane of $100 \mathrm{kDa}$ cut-off. The slurry obtained was freeze-dried and used for X-ray diffraction analysis. The radiation source used was Ni-filtered, Cu-Ka. The voltage was set to $40 \mathrm{kV}$ and the current to $40 \mathrm{~mA}$. Samples were mounted on a sample holder and scanned from $5^{\circ}$ to $60^{\circ}$ in $2 \theta$ at a rate of $5 \mathrm{~min}^{-1}$ with 0.04 degree per step with a LynxEye linear position sensitive detector with $2.94^{\circ}$ coverage in $2 \theta$. This was recorded on a Bruker D8 Advance fitted with a robotic sample changer.

Scanning Electron Microscopy (SEM). Images were recorded on an FEI Quanta 200 field emission scanning electron microscope operated at an accelerating voltage of $10 \mathrm{kV}$. Samples were prepared on silicon slides. The slides were cleaned by exposure to piranha solution (70:30 v/v sulfuric acid/hydrogen peroxide), washed with MQ water and ethanol and dried with nitrogen gas. (Caution! Piranha solution is highly corrosive. Extreme care should be taken when handling piranha solution and only small quantities should be prepared.). Following this, $2 \mu \mathrm{L}$ of a concentrated suspension of RPs in $50 \mathrm{mg} \mathrm{mL}^{-1} \alpha \mathrm{CD}$ were spotted and immediately blotted and then left to air-dry. A thin layer of gold ( $\sim \mathrm{nm})$ was deposited on the sample by sputter-coating. 


\section{RESULTS AND DISCUSSION}

Formation and Characterization of PPRX Replica Particles and Hollow Capsules. Fabrication of the PPRX replica particles or capsules begins with adsorption of di-hydroxy PEG $\left(M_{\mathrm{w}}=3-20 \mathrm{kDa}, 2 \mathrm{mg} \mathrm{mL}^{-1}\right.$ in MQ water) onto mesoporous (5 $\mu \mathrm{m}$, pore size $50 \mathrm{~nm}, 2$

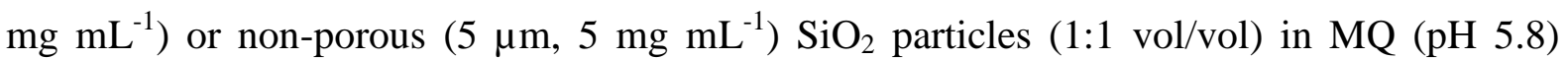
(Scheme 1, step a). Thermal gravimetric analysis (TGA) of the PEG-loaded templates revealed that by mass, $\mathrm{ca} .16 \%$ and $3 \%$ consisted of PEG physically adsorbed onto the mesoporous and non-porous $\mathrm{SiO}_{2}$ templates, respectively (Supporting Information, Figure S1). The physical adsorption of PEG onto a surface can be described by the loop-train-tail model where: the trains consist of segments in direct contact with the surface; the loops have no direct contact with the surface but are in close proximity; and the tails are the nonadsorbed chain ends. ${ }^{44}$ Thus, when the $\mathrm{SiO}_{2}$ particles are resuspended into a concentrated $\alpha \mathrm{CD}$ solution (100 $\mathrm{mg} \mathrm{mL}^{-1}$ ), the $\alpha \mathrm{CDs}$ are able to thread onto the adsorbed PEG chains via the PEG tails (Scheme 1, step b). The inclusion of $\alpha \mathrm{CD}$ onto PEG physically absorbed on $\mathrm{SiO}_{2}$ particles has been previously used to create bulk hydrogels. ${ }^{44}$, 45 These studies suggested, when the PEG $M_{\mathrm{w}}$ is low ( $\left.\leq 2 \mathrm{kDa}\right)$, complete desorption of the PEG chains from the $\mathrm{SiO}_{2}$ templates occurs. ${ }^{44}$ This is attributed to complete inclusion of $\mathrm{CD}$ resulting in crystalline adducts, and suggests that the forces driving complexation are higher than the interaction of PEG with the $\mathrm{SiO}_{2}$ surface. However, when PEG $M_{\mathrm{w}}$ increases (6 $\mathrm{kDa}$ ), physical gelation occurs where there is a balance between (i) PEG chains remaining physically adsorbed onto the colloidal particles, and (ii) there is sufficient CD threading, and hence hydrogen bonding within and between PEG chains. ${ }^{44}$ Also, studies revealed when $[\alpha \mathrm{CD}] /[\mathrm{PEG}] \geq 5$, gelation occurs. Whereas when this ratio is $\leq 5$, but $\leq 2$, soluble solutions are achieved. ${ }^{44}$ It should be noted, in these studies, ${ }^{44}$ non-porous $\mathrm{SiO}_{2}$ templates have significantly smaller diameters (i.e., $12 \mathrm{~nm}$ ) than the templates we utilize in this study (i.e., 5 
$\mu \mathrm{m})$. Thus, major differences in template surface areas should be kept in mind when comparing this study and those in literature. Nevertheless, to prevent complete desorption or physical gel formation, PEG with $\mathrm{M}_{\mathrm{w}}$ of $3.35 \mathrm{kDa}$ was predominately used, while a $[\alpha \mathrm{CD}] \geq$ $100 \mathrm{mg} \mathrm{mL}^{-1}$ was used throughout this study. At this condition, a high degree of $\alpha \mathrm{CD}$ threading (> 90\%, $34 \alpha$ CDs per PEG, Supporting Information, Figure S2) was achieved on the surface-adsorbed PEGs while avoiding desorption. After constantly agitating this suspension, discrete surface-confined PPRX particles were obtained. This is attributed to hydrogen bonding between the threaded $\alpha \mathrm{CDs}$ resulting in crystalline $\alpha \mathrm{CD}$ bundled domains. XRD analysis confirmed these conformational changes, where reflection lines at $2 \theta=19.9$ appeared in all cases when CD and PEG were present (Supporting Information, Figure S3). This characteristic peak is attributed to the formation of CD channel structures via CD/PEG inclusion complexation. ${ }^{46}$ When the inorganic supporting substrates were dissolved with hydrofluoric acid (HF) in $[\alpha \mathrm{CD}]$ is $50 \mathrm{mg} \mathrm{mL}^{-1}$, stable replica particles (ca. $4.7 \mu \mathrm{m} \pm 0.5$ ) and hollow capsules (ca. $5.5 \mu \mathrm{m} \pm 0.4$ ) were observed in the presence of $\alpha \mathrm{CD}$, as confirmed through differential interference microscopy (DIC) and scanning electron microscopy (SEM) (Figure 1a,c and Figure 1b,d, respectively). The capsule thickness was observed to be $c a .50$ nm via SEM (Figure 2d). Interestingly, the stable particles did not undergo any shrinkage or swelling, thus suggesting that the $\alpha \mathrm{CD} / \mathrm{PEG}$ complexes form a rigid architecture. This is contrary to pure PEG particles, which are generally soft and highly deformable upon template removal. ${ }^{47}$ SEM images of the replica particles revealed a rougher topology than the hollow capsules (Figure 1c and d, respectively). This may be due to the longer complexation time allowed between $\alpha \mathrm{CD}$ and PEG for capsules (7 d and $12 \mathrm{~h}$ for capsules and replica particles, respectively). It is noted that a longer $\alpha \mathrm{CD}$ threading time for the capsules was necessary to avoid fragmentation of the capsules upon dissolution of the template. This further suggests that a high threading degree coupled with a more packed arrangement of PPRXs is required 
to obtain stable, hollow spherical films. The fabrication of smaller particles $(\leq 300 \mathrm{~nm})$ has not been explored in this study. However, it is believed fine tuning the method would be required as a relatively high thickness (Figure 1) is required to maintain stability.

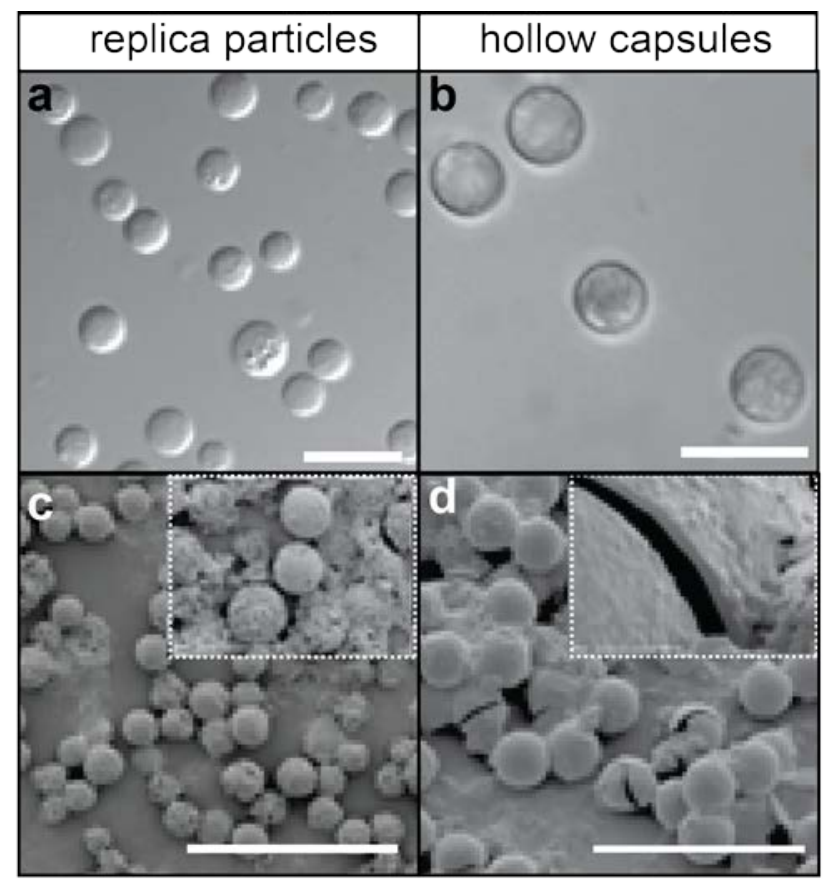

Figure 1. DIC images of (a) replica particles and (b) hollow capsules. Scale bars are $10 \mu \mathrm{m}$. SEM images of (c) replica particles and (d) and hollow capsules with higher magnification insets showing particles roughness and capsule thickness, respectively. Scale bars are $20 \mu \mathrm{m}$. Note, linear di-hydroxyl PEG of $M_{W} 3.35 \mathrm{kDa}$ was used in both instances.

(i) We initially explored the effect of varying the $M_{\mathrm{w}}$ of linear di-hydroxyl PEG (i.e., 1.10, 3.35, 6, 10 and $100 \mathrm{kDa}$ ) in terms of particle and capsule formation. Stable hollow capsules with approximately the same size could be fabricated using $M_{\mathrm{w}}$ of 3.35, 6 and $10 \mathrm{kDa}$ (SI, Figure S4a-c). However, when 100 kDa PEG was used, after 12 h of incubation, only debris could be observed after core dissolution. This result is most likely due to a decrease in complex formation and is mostly likely attributed to: (i) an increase in "train" segments where PEG chains are in direct contact with the $\mathrm{SiO}_{2}$ surface, and thus cannot be easily 
disrupted; and (ii) a decrease in the surface density of PEG tails, which is necessary for $\alpha \mathrm{CD}$ threading. When $1.10 \mathrm{kDa}$ PEG was used hollow capsules could not be formed. This result is attributed to the low $M_{\mathrm{w}}$ of PEG, which results in a significantly smaller number of trains and a high threading degree of $\alpha \mathrm{CD}$, further promoting displacement of the adsorbed PEG. This is further supported by the fact that replica particles formed from 1.10 kDa PEG did not retain the spherical shape of the template and formed self-collapsed structures (Supporting Information, Figure S5). Stable replica particles could be formed from PEG with $M_{\mathrm{w}}$ ranging from 3.35-100 kDa (data not shown for PEGs with $M_{\mathrm{w}} 6$ and $100 \mathrm{kDa}$ ). For particles prepared using $3.35 \mathrm{kDa}$ PEG, the hydrodynamic thickness and adsorbed mass density on $\mathrm{SiO}_{2}$ can be extrapolated from the literature to be $c a .1 .28 \mathrm{~nm}$ and $\sim 0.23 \mathrm{mg} \mathrm{m}^{-2}$, respectively. ${ }^{48}$ The adsorbed thickness is close to the Stokes radius $\left(R_{h}\right)$ of this specific PEG chain $(\sim 1.9 \mathrm{~nm}){ }^{49}$ However, for PEGs larger than $3.35 \mathrm{kDa}$, the discrepancy between $R_{h}$ of the PEG and the adsorbed thickness increases. This further highlights that the larger $M_{\mathrm{w}} \mathrm{PEG}$ chains are more spread over the surface with a smaller "tail" density over which the $\alpha$ CDs can thread. ${ }^{49}$ Furthermore, when the $3.35 \mathrm{kDa}$ PEG adsorption time was decreased to 15 min (as opposed to $12 \mathrm{~h}$ ), the resulting capsules were destabilized upon template removal, forming shell debris (Supporting Information, Figure S6). At this PEG $M_{w}$, adsorption reaches near saturation within $4 \mathrm{~min}$; however, over longer time scales it is suggested that the adsorbed chains undergo conformational transitions. Thus, this result that is also in accordance with other reports, ${ }^{44,45}$ suggesting: (i) an optimal range of PEG $M_{\mathrm{w}}$ is required to allow for sufficient and stable PEG adsorption (does not completely desorb from the surface when $\alpha \mathrm{CDs}$ are added); and (ii) longer adsorption times are necessary to allow the adsorbed PEG chains to undergo conformational changes required for a more stable configuration, preventing desorption of the PEG chain upon $\alpha \mathrm{CD}$ threading and core dissolution. Additionally stable hollow capsules were not produced when 100 kDa PEG was used. This is likely because the overall PEG "tail" 
density reduces as $M_{\mathrm{w}}$ increases, and importantly, the overall surface coverage available for non-porous templates is substantially lower than mesoporous templates, thus the effective amount of cross-linking/stabilization sites is reduced.

Disassembly of the PPRX Particles. As the PPRX particles are not covalently stabilized by bulky end groups or covalent bridges, disassembly of the particles can be achieved by diluting the $[\alpha \mathrm{CD}]$ in the supernatant. PPRX formation and disassembly kinetics are dependent on $[\alpha \mathrm{CDs}]$ in bulk, with the rate of threading/de-threading being proportional to $[\alpha \mathrm{CD}]{ }^{35}$ Hence, we investigated PPRX particle degradation as a function of $[\alpha \mathrm{CD}]$. Individual solutions of $\alpha \mathrm{CD}$ and PEG are clear, even at high concentrations. However, upon mixing the two solutions (where the $[\alpha \mathrm{CD}] \geq 44 \mathrm{mg} \mathrm{mL}$ ), turbidity occurs due to crystallization of the inclusion complexes. ${ }^{50,51}$ Therefore, disassembly of the PPRX particles was monitored by measuring the non-specific absorbance at $500 \mathrm{~nm}$ by optical microscopy. A decrease in absorbance was observed when the $[\alpha \mathrm{CD}]$ was $10 \mathrm{mg} \mathrm{mL}^{-1}$ and $5 \mathrm{mg} \mathrm{mL}^{-1}$. Noteworthy, this decrease in value stabilized after $10 \mathrm{~min}$ and no significant decrease in the absorbance was observed over longer times (Figure 2). Furthermore, intact discrete particles could still be observed in the DIC images, although the particles seemed rougher than those with a surrounding $[\alpha \mathrm{CD}]$ of $50 \mathrm{mg} \mathrm{mL}^{-1}$ (Figure 2, insert). This may suggest that at $[\alpha \mathrm{CD}]=$ 10 or $5 \mathrm{mg} \mathrm{mL}^{-1}$, partial de-threading occurs via the release of some $\alpha \mathrm{CDs}$ from the particles to the bulk. Near complete particle degradation was observed at substantially lower $\alpha \mathrm{CD}$ concentrations ( $\leq 1 \mathrm{mg} \mathrm{mL}^{-1}$ ), as confirmed by an almost zero absorbance as well as optical microscopy (Figure 2, insert). Noteworthy, the particles are stable in the presence of high $[\alpha \mathrm{CD}]$ of $50 \mathrm{mg} \mathrm{mL} \mathrm{m}^{-1}$, thus it is expected dissociation of PPRXs from the particles is minimal. Nevertheless, as the $\alpha \mathrm{CDs}$ are excluded from individual PPRXs during degradation (lowering $[\alpha C D]$ ) it is likely that there is a minimum threshold where individual PPRXs with a low threading degree are dissociated due to highly reduced H-bonding potential. 

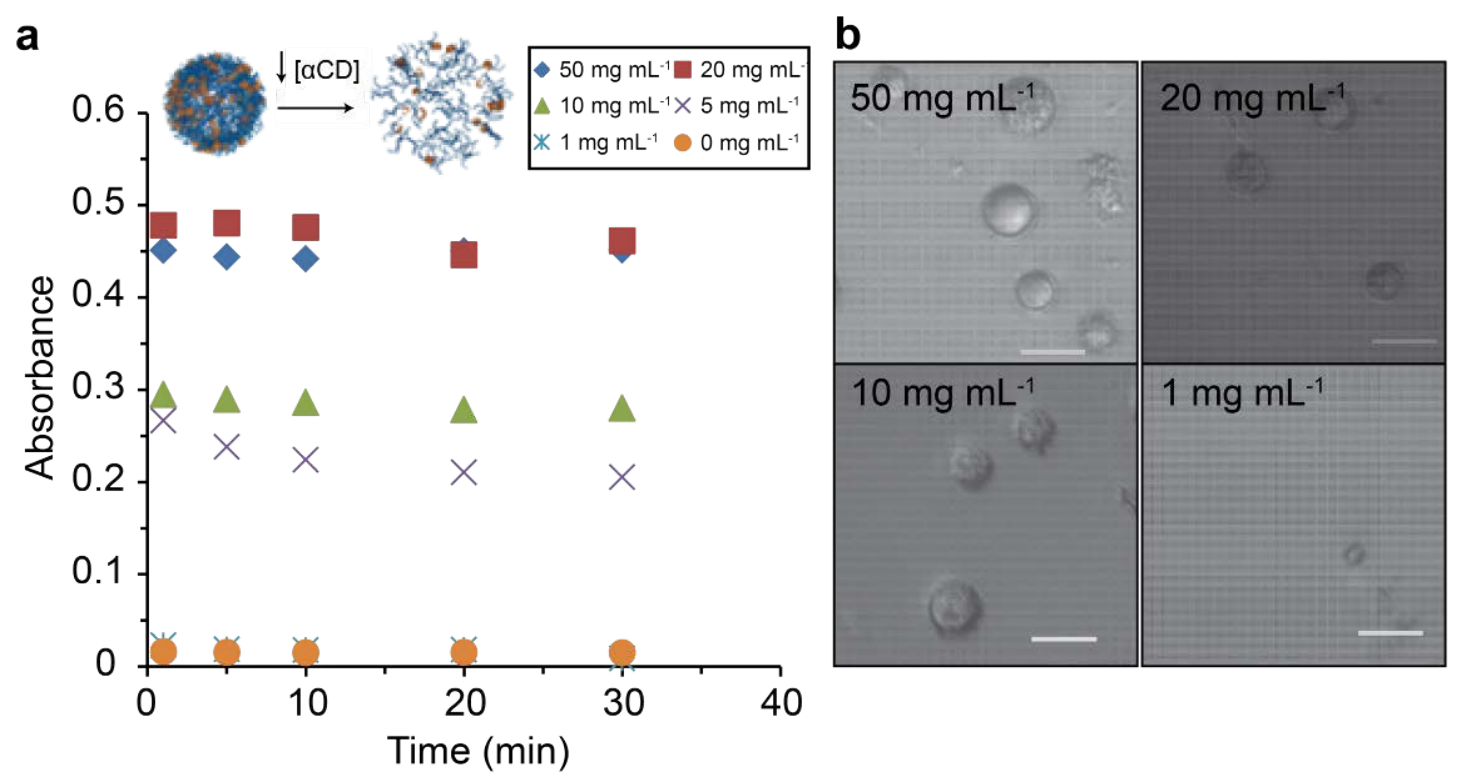

Figure 2. $\left[\alpha \mathrm{CD}\right.$ ]-dependent degradation of replica particles formed from PEG of $M_{W} 3.35$ $\mathrm{kDa}$, as measured by (a) UV-vis spectroscopy at $500 \mathrm{~nm}$. (b) DIC images of the replica particles at $30 \mathrm{~min}$. Scale bars are all $10 \mu \mathrm{m}$.

To reduce the rate of CD dethreading, a semi-blocking strategy can be utilized, where bulky end groups with varying molecular dimensions can be covalently attached to the ends of the PEG chain. ${ }^{52,53}$ This concept has been shown for $\beta C D / P E G$ complexes where PEG chain ends were coupled with bulky end groups of varying size. ${ }^{54}$ The solid state cavity diameter of $\alpha \mathrm{CD}$ is $0.57 \mathrm{~nm}{ }^{55}$ therefore by functionalizing PEG ends with bulky groups only slightly larger than the cavity of $\alpha \mathrm{CD}$, the rate of PPRX particle formation and degradation can be controlled. To explore this, a set of PEGs functionalized with different semi-blocking groups were synthesized (See Supporting Information for synthetic protocol) and preliminary studies on PPRXs formation and degradation kinetics were carried out in the absence of $\mathrm{SiO}_{2}$ templates. It should be noted, although end-group substitution with bulky end groups was 4098\%, CD inclusion complexation with $\mathrm{R}_{1}$ (homo bifunctional hydroxyl PEG) occurs via both 
ends. Thus, the rate of $C D$ complexation through the $\mathrm{R}_{2}-\mathrm{R}_{7}$ (PEG with substituted ends) will be reduced, thereby affecting particle formation and disassembly behavior. Briefly, PPRX formation was achieved by mixing a $0.14 \mathrm{M}$ solution of $\alpha \mathrm{CD}$ with a $0.6 \mu \mathrm{M}$ solution of semiblocked PEGs $\left(\mathrm{R}_{1}-\mathrm{R}_{7}, M_{W} \mathrm{PEG}=3.35 \mathrm{kDa}\right)$. For the degradation kinetics, the formed PPRXs were freeze dried, and subsequently rehydrated in MQ water (2 mL). Both formation and degradation kinetics were monitored by measuring the solution absorbance at $500 \mathrm{~nm}$ over 50 min. It is noted that linear di-hydroxy PEG $\left(\mathrm{R}_{1}\right)$ is one of the most commonly used PEGs for $\alpha \mathrm{CD}$ inclusion, and was used in the first part of this study. It was found that $\alpha \mathrm{CD}$ was able to thread onto $\mathrm{R}_{1}-\mathrm{R}_{7}$, and importantly, the degradation rate could be tuned (Supporting Information, Figure S7). It should also be noted that the formation of PPRX with $\mathrm{R}_{4}$ was not monitored by UV-vis spectroscopy, as a supersaturated solution $\left([\alpha \mathrm{CD}]=c a .210 \mathrm{mg} \mathrm{mL}^{-1}\right)$ was required for observable absorbance values; therefore results could not be compared with the other semi-blocking groups. $\mathrm{R}_{1}-\mathrm{R}_{5}$ was chosen for further studies, as they represent a diverse range of degradation rates.

Formation of PPRX replica particles using semi-blocked PEGs $\left(R_{2}-R_{5}\right)$ with mesoporous $\mathrm{SiO}_{2}$ templates took a considerably longer time when compared with di-hydroxy PEG, $\mathrm{R}_{1}$ (> 2 weeks as opposed to $12 \mathrm{~h}$, respectively). The formed replicas all mirrored the template particles in size and shape (Figure 3a-c). When DMSO was added, instant degradation of the replicas occurred, resulting in clear solutions. This confirms the complete removal of the template and the reversible nature of PPRXs. XRD analysis further confirmed that for all of the particles, characteristic channel-type crystalline structures associated with PEG/ $\alpha \mathrm{CD}$ assembly at $2 \theta=11.5$ and 19.7 were observed, which is in agreement with literature. ${ }^{56}$ It was also noted that a lower yield of PPRX particles was obtained. Differences in yield may be attributed to the lower final threading degree due to a high energy barrier when using semi-blocked PEGs. Interestingly, this did not affect the replica particle size and 
thus suggests that complete threading is not required to obtain stable replica particles with good morphology (Figure 3a and b, respectively). The degradation profile of the formed PPRX particles using $\mathrm{R}_{1}-\mathrm{R}_{5}$ was also monitored by an adapted turbidity method monitoring light scattering intensity, ${ }^{57}$ where a decrease in intensity translates to the presence of less PPRXs particles in bulk. For the degradation curves to be comparable, a final $[\alpha \mathrm{CD}]$ of $\sim 2.5$ $\mathrm{mg} \mathrm{mL}^{-1}$ was chosen as well as an appropriate content in particles to obtain similar initial light scattering intensities, $\mathrm{t}_{0}$. Significant differences in degradation kinetics were observed depending on the semi-blocking group, with a decreasing degradation speed following the order $\mathrm{R}_{1}>\mathrm{R}_{2}>\mathrm{R}_{5}>\mathrm{R}_{3}>\mathrm{R}_{4}$ (Figure 3c). Half intensity was reached after $3 \mathrm{~h}$ for particles formed using $\mathrm{R}_{4}$ functionalized semi-blockers while degradation only took a few seconds using $\mathrm{R}_{1}$ or $\mathrm{R}_{2}$ (Figure 3c). Noteworthy, the decay for PEG $\mathrm{R}_{4}$ (Figure 3c) continued for several hours following the time frame reported herein (data not shown). The slowed degradation is possibly the result of an equilibrium slowly reached from the $\alpha \mathrm{CDs}$ excluded from the particles upon degradation. These results highlight the advantage of this approach to engineer the PPRX particles with tunable degradation kinetics.

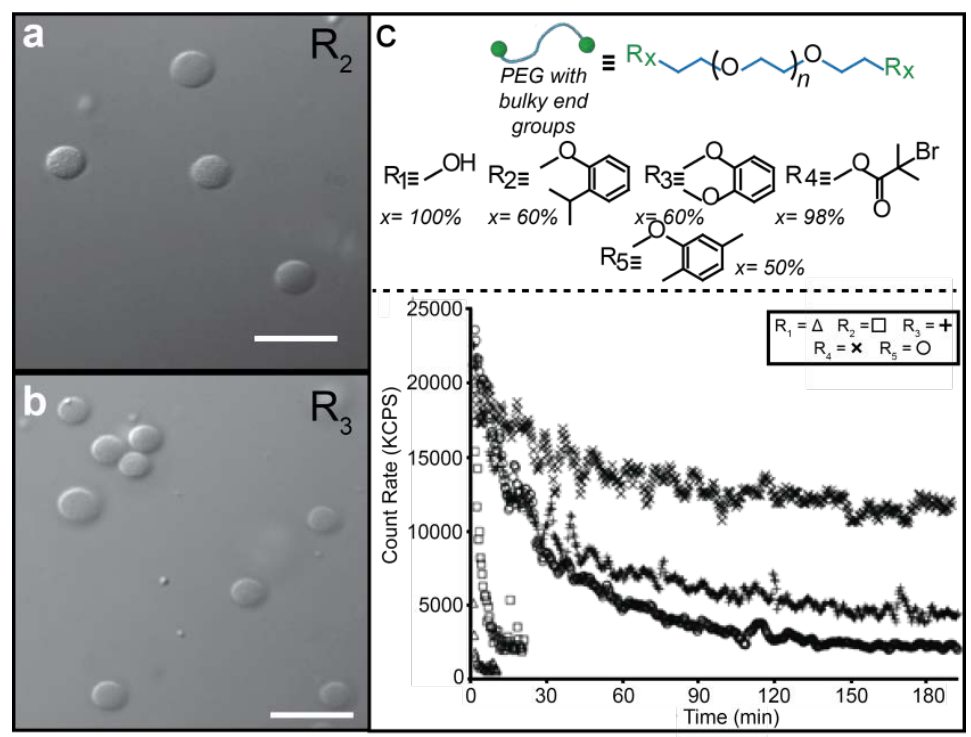


Figure 3. DIC images of replica particles obtained from semi-blocked PEGs using (a) R2 and (b) R3 after core dissolution. (c) Graphical representation of the semi-blocked PEGs $\left(\mathrm{R}_{1}-\mathrm{R}_{5}\right)$ used to form transiently stabilized RPs and their corresponding degradation profile, as monitored by scattering intensity. Scale bars are $10 \mu \mathrm{m}$. Note $\mathrm{x}$ corresponds to the \% end group substitution.

Covalent Stabilization of PPRX Particles. To demonstrate the versatility of our method, covalent stabilization on the PPRX particles was also conducted. In this process, particles were formed using the same strategy described in Scheme 1 with amine end-terminated multi-arm PEG instead of linear PEG. Specifically, 4- and 8-arm PEGs of $M_{w} 40$ and $10 \mathrm{kDa}$ respectively, were used. The presence of amine end-groups allows for the cross-linking of the PPRX particles with glutaraldehyde (Scheme 2). The particles were found to be stable in the absence of $\alpha \mathrm{CD}$ after template removal and/or after addition of DMSO, thus suggesting successful stabilization of the particles via covalent bridges. 


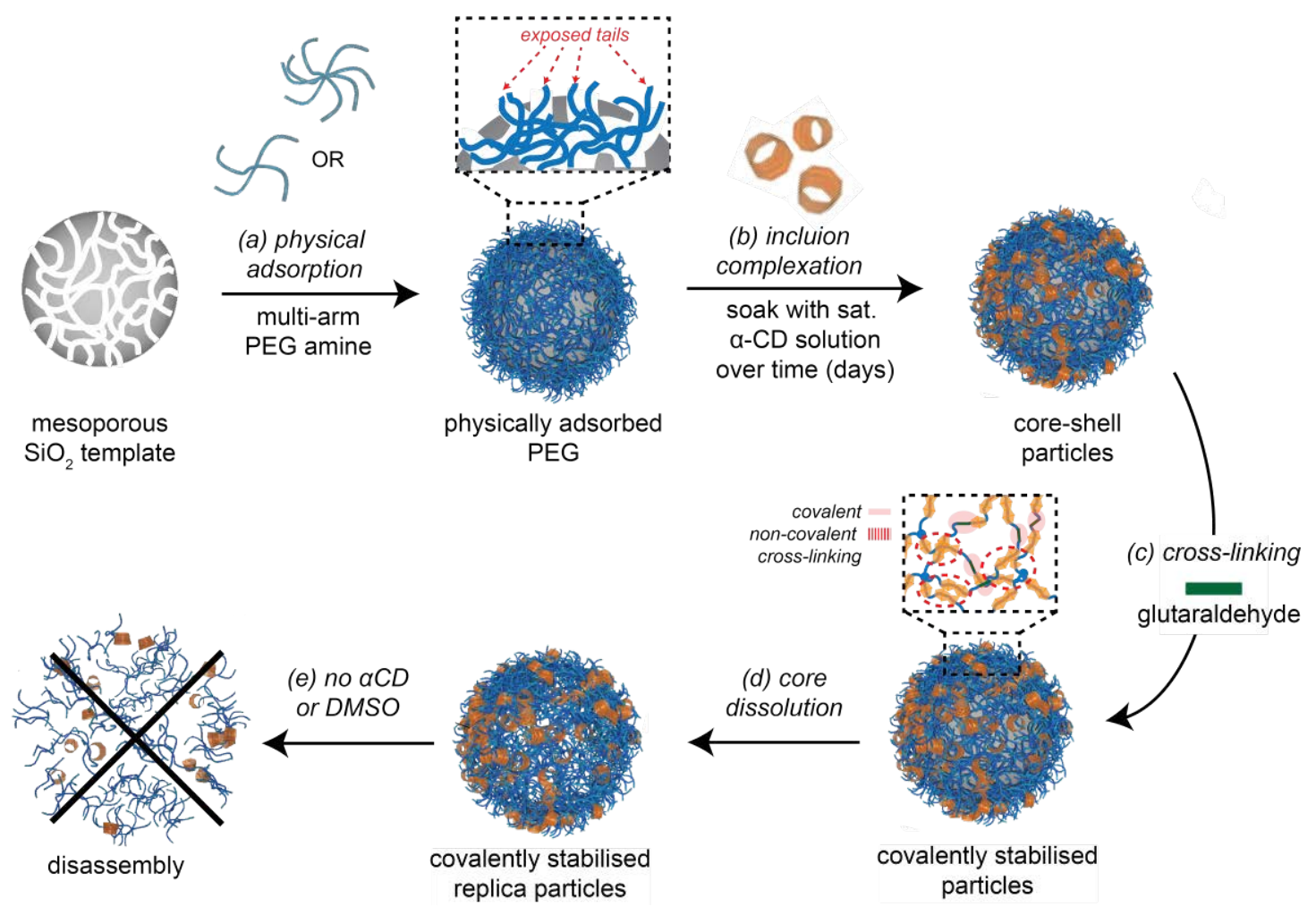

Scheme 2. Formation of covalently cross-linked PRX particles by (a) first adsorbing 4- or 8arm PEG amine onto mesoporous $\mathrm{SiO}_{2}$ templates followed by (b) suspending the formed construct in a saturated solution of $\alpha \mathrm{CDs}$ for $12 \mathrm{~h}$. (c) Covalent stabilization is achieved via the addition of glutaraldehyde, resulting in covalent bridges between the PEG chain ends. (d) After core dissolution, replica particles, stabilized by covalent bridges and CD rich crystalline domains, are formed. (e) Upon application of DMSO or MQ water in the absence of $\alpha \mathrm{CD}$, disassembly was prevented, thus confirming successful covalent stabilization.

No substantial particle swelling was observed in pure MQ water for both 4- and 8-arm PEGs (Figure $4 \mathrm{a}$ and $4 \mathrm{c}$, respectively). This suggests inclusion of $\alpha \mathrm{CD}$ remained intact during the cross-linking procedure, because particles composed solely of multi-armed PEGs using the same template swelled considerably after template removal. ${ }^{47}$ However, a slightly smaller size of the particles compared to the templates $(5 \mu \mathrm{m})$ in the case of 4-arm PEG was observed. 
This could be due to the presence of both covalent crosslinks and hydrophobic $\alpha \mathrm{CD}$ mesocrystalline domains bundling together, making the particles shrink after template removal. Similar trends have also previously been observed for PEG/ $\alpha \mathrm{CD}$ hydrogels. ${ }^{58}$ The inclusion of $\alpha \mathrm{CD}$ can be also confirmed by the $\mathrm{pH}$ responsive behavior of the particles; primary $-\mathrm{OH}$ groups of the threaded $\alpha \mathrm{CD}$ are deprotonated at higher $\mathrm{pH}$, leading to a swelled PRX structure when compared with the neutral pH of 5.8 (Figure 4). Interestingly, after the first cycle of swelling/shrinkage, the size observed on shrinkage is higher than the initial size and is still stable on the following cycles (Supporting Information, Figure S8). This is likely to be attributed to $\mathrm{CD}$ rich pockets being released and reverting to a more suitable conformation upon shrinkage. Particles formed using 8-arm PEG of $M_{w} 10 \mathrm{kDa}$ (1.3 kDa per arm) are expected to have a much higher threading degree of $\alpha \mathrm{CD}$ than particles formed using 4-arm PEG of $M_{w} 40 \mathrm{kDa}$ (10 kDa per arm) because larger PEGs generally lead to a lower threading degree for linear PRXs. ${ }^{35}$ Therefore, due to swelling, larger particle sizes can be expected (and were observed) when using the higher $M_{w}$ multi-arm PEG (Figure 4c,d versus $4 a, b$, respectively). A lower final amount of $\alpha \mathrm{CD}$ in the particles would result in a more hydrated PEG-like matrix, leading to a more swollen structure. This study thus shows that by careful selection of PEG, end group functionality, and $M_{\mathrm{w}}$, covalent stabilization and particle swellability can be easily tuned. 


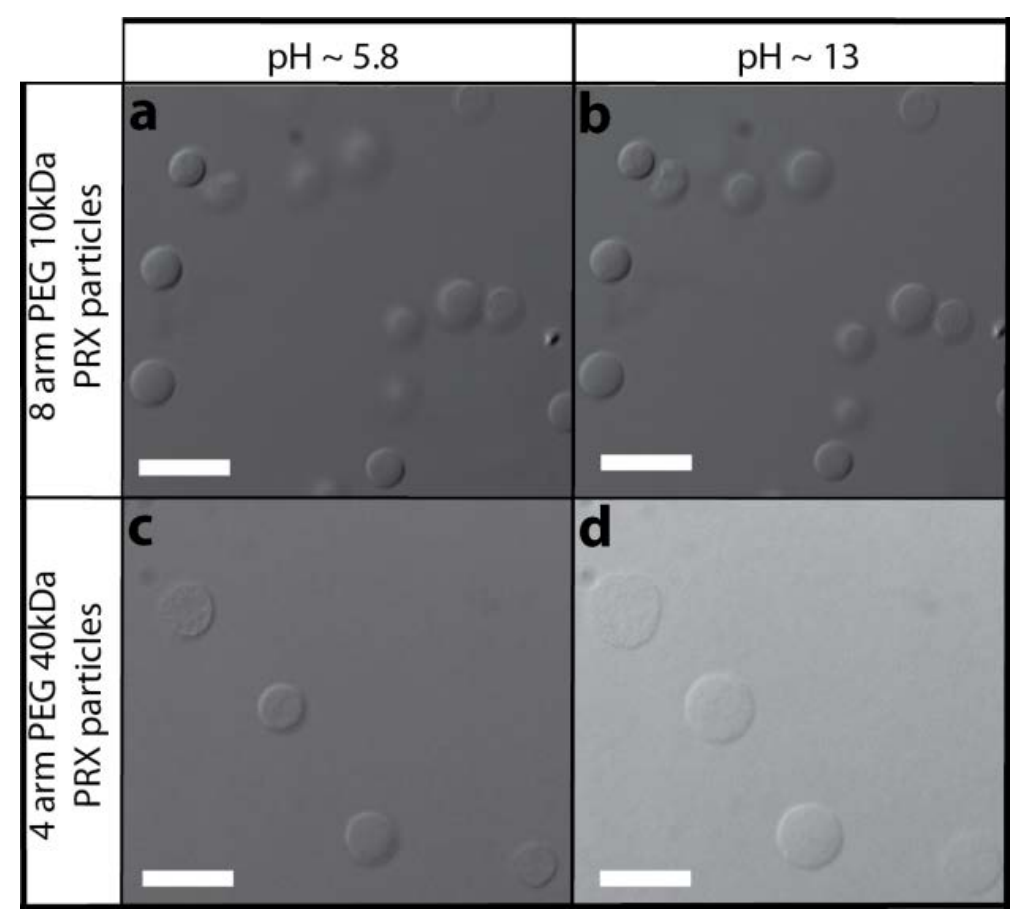

Figure 4. DIC images of covalently stabilized particles using 8-arm PEG of $M_{W} 10 \mathrm{kDa}$ at (a) pH 5.8 and (b) pH 13, and using 4 arm PEG of $M_{W} 40 \mathrm{kDa}$ at (c) pH 5.8 and (d) pH 13. Scale bars are $5 \mu \mathrm{m}$.

Loading and Release of Model Compounds from PPRX Particles. To further explore the application of these materials, fluorescent drugs were chosen as model compounds to qualitatively assess loading and release of small molecules from PPRX particles using PEG $\mathrm{R}_{1}$. Briefly, doxorubicin (DOX) (100 $\mathrm{mg} \mathrm{mL}^{-1}$ in DMSO) or an alkyl-modified Rhodamine (RhD-C) (10 $\mathrm{mg} \mathrm{mL}^{-1}$ in DMSO) were loaded into PPRX replica particles while suspended in a $0.1 \mathrm{M}$ solution of $\alpha \mathrm{CD}$. It should be noted that a large excess of dye was used in the preparation of the particles to ensure significant loading. Additionally, for both DOX and RhD-C, a significant proportion of the cargo was released upon template removal and during the following washing steps. Successful loading of RhD-C and DOX could be achieved, as observed by fluorescence microscopy (Figure 5, insert), and from $\mathrm{T}_{0}$ signals after complete 
degradation of the particles $\left(1.85 \times 10^{6}\right.$ fluorescence unit (FU) for RhD-C and $0.325 \times 10^{6}$ (FU) for DOX, respectively). The release of DOX was observed even when the particles were not degraded (Figure 5a, triangles). Upon particle degradation (decrease in $[\alpha C D]$ ), a significant release of DOX was further observed (ca. 60\% more, Figure 5a, triangles versus crosses). The non-specific release of DOX is attributed to poor entrapment within the PPRX matrix. We note that the degradation of the PPRX RPs was significantly retarded by the presence of DOX (i.e., several hours, Figure 5a), as at equal $[\alpha \mathrm{CD}]$, PPRXs particles degraded in several seconds (Figure 3). This is likely due to DOX dissolution limits at the $\mathrm{pH}$ of PBS (pH 7.4). Thus, in this given scale, even if $\alpha \mathrm{CD}$ dethreading is fast, it is prevented by hydrophobic pockets of, slowly dissolving, DOX. Similar release profiles of physically adsorbed DOX were also observed when using other capsule materials but to a lesser extent. $^{59}$ In contrast, no release of RhD-C from PPRX particles was observed when the loaded particles were not degraded (Figure 5b, circles). Also, when release was initiated by particle degradation, the retardation of degradation was not as pronounced as with DOX (Figure 5b, triangles versus Figure 5a, triangles, respectively). These results suggest that the cargo has a significant impact on the degradation kinetics. Furthermore, the cargo release kinetics can be controlled by utilizing semi-blocked PEGs. The use of PEG $\mathrm{R}_{3}$ slowed the release kinetics of RhD-C when compared with di-hydroxy PEGs (Figure 5b). Additionally, when PPRX particles were assembled using PEG $\mathrm{R}_{4}$ and loaded with $\mathrm{RhD}-\mathrm{C}$, no degradation or release was observed over the first few hours after substantially lowering the $[\alpha \mathrm{CD}]$ (Supporting Information, Figure S9). Overall, PPRX particles showed more controlled release profiles of RhD-C. This could be due to the better integration of RhD-C into the particle matrix, thus reducing the extent of non-specific release. These qualitative results suggest that these particles may be used for the sequestration and subsequent release of cargo can be tailored. Loading and release studies are used as a proof-of-concept showing the 
formed particles can encapsulate and release cargo, hence loading capacities and efficiencies were not evaluated.
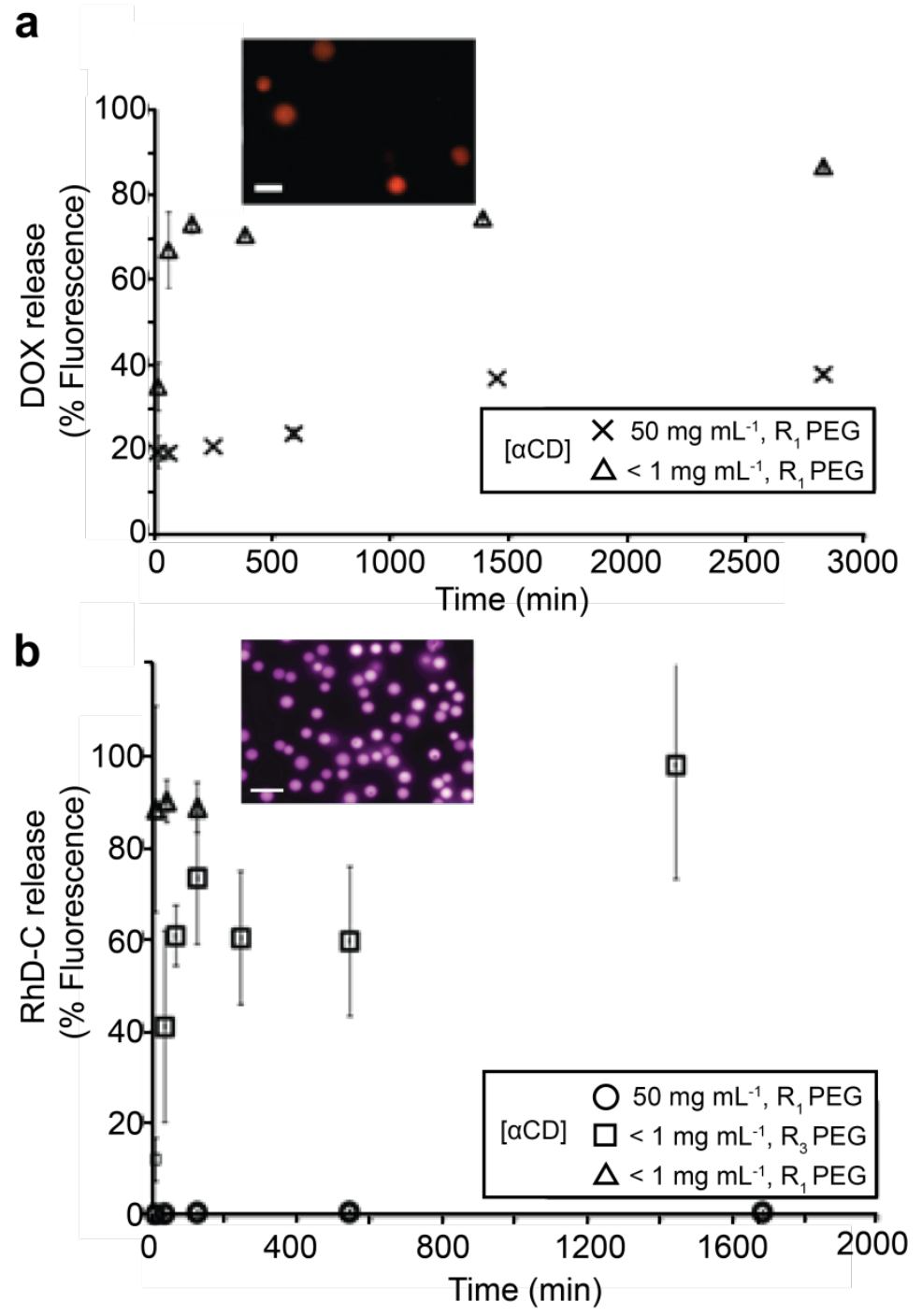

Figure 5. Release and retention profiles of (a) DOX-loaded particles at low and high [ $\alpha \mathrm{CD}]$ and of (b) RhD-C loaded particles at low and high concentrations of $\alpha \mathrm{CDs}$ in solution when PEG $\mathrm{R}_{1}$ or $\mathrm{R}_{3}$ are used. Inserts are fluorescence microscopy images of the cargo-loaded replica particles. Scale bars are 5 and $10 \mu \mathrm{m}$, respectively.

\section{CONCLUSIONS}


In this study, a facile, one-pot methodology, utilizing template assembly and CD host-guest chemistry, was developed to form PPRX particles and capsules. Using this method, the steric hindrance of CDs and PRXs, which typically limit their use in the microscopic domain, can be overcome, resulting in PPRX materials with highly tunable characteristics. In this process, PEG is initially pre-adsorbed onto a silicon oxide surface and the construct is subsequently suspended in a concentrated $\alpha \mathrm{CD}$ in solution. This drives $\mathrm{CDs}$ complexation with the physically absorbed PEG chains through the exposed tails. Upon continuous agitation, crystalline domains attributed to CD hydrogen-bonding are formed resulting in stable, homogenous, PPRX particles and capsules. By simply changing the end group functionality of the PEG chains or the PEG $M_{\mathrm{w}}$, the rate of particle formation and degradation could be finely tuned. In tailoring these factors, control over cargo release profiles could be achieved, as shown using various model drugs. Furthermore, covalent stabilization of the particles was also shown to be easily achievable by the addition of small cross-linking molecules. This resulted in permanent bridges between individual PPRXs, thus creating PRX particles. The reported methodology provides a simple and versatile platform to generate PPRX nanoscale materials with high tunability, which to our knowledge, cannot be easily achieved using current conventional systems, where pre-synthesis and various isolation and purification steps are necessary. Structurally, PPRX-based materials allow for increased tunability, which cannot be easily achieved by their covalent counterparts. For example, the rigidity of PPRXbased materials which were shown to affect degradability and cargo release can be controlled by simply altering the CD threading degree, judicious selection of bulky end-groups, or by tuning the cross-linking degree. On the contrary, for completely covalent analogues, tuning the cross-linking degree is considered the main avenue towards altering particle or capsule permeability, aside from material selection, which can also be applied in PPRX systems. Due to these advantages and the results presented in this study, it is anticipated that this new 
technique will open new avenues for PPRXs and PRXs-based technologies, where often steric hindrance of the CDs plays a great inhibitory role.

\section{ASSOCIATED CONTENT}

\section{Supporting Information}

Experimental procedure for PEG $\mathrm{R}_{1}-\mathrm{R}_{7},{ }^{1} \mathrm{H}$ NMR spectra, XRD diffractograms, TGA profiles, UV-Vis formation and degradation kinetics, DLS size measurements, fluorescence intensity of cargo, and DIC and SEM images of the particles and hollow capsules (PDF). This materials is available free of charge via the Internet at http://pubs.acs.org.

\section{AUTHOR INFORMATION}

\section{Corresponding Author}

*E-mail: fcaruso@unimelb.edu.au.

\section{Author Contributions}

The manuscript was written through contributions of all authors. All authors have given approval to the final version of the manuscript.

$\ddagger$ These authors contributed equally.

\section{Notes}

The authors declare no competing financial interest.

\section{ACKNOWLEDGMENTS}

This research was in part conducted and funded by the Australian Research Council (ARC) Centre of Excellence in Convergent Bio-Nano Science and Technology (project number CE140100036), and the ARC under the Australian Laureate Fellowship (FL120100030, F.C.). This work was performed in part at the Materials Characterisation and Fabrication Platform 
(MCFP) at the University of Melbourne and the Victorian Node of the Australian National Fabrication Facility (ANFF). The authors would like to thank Dr. Yi Ju and Farshad Motevalizadeh for their assistance.

\section{REFERENCES}

1. $\quad$ Esser-Kahn, A. P.; Odom, S. A.; Sottos, N. R.; White, S. R.; Moore, J. S., Triggered release from polymer capsules. Macromolecules 2011, 44, 5539-5553.

2. Stuart, M. A. C.; Huck, W. T.; Genzer, J.; Müller, M.; Ober, C.; Stamm, M.; Sukhorukov, G. B.; Szleifer, I.; Tsukruk, V. V.; Urban, M., Emerging applications of stimuliresponsive polymer materials. Nature Mater. 2010, 9, 101-113.

3. Wang, Y.; Hosta-Rigau, L.; Lomas, H.; Caruso, F., Nanostructured polymer assemblies formed at interfaces: applications from immobilization and encapsulation to stimuli-responsive release. Phys. Chem. Chem. Phys. 2011, 13, 4782-4801.

4. Delcea, M.; Möhwald, H.; Skirtach, A. G., Stimuli-responsive LbL capsules and nanoshells for drug delivery. Adv. Drug Deliv.Rev. 2011, 63, 730-747.

5. Pack, D. W.; Hoffman, A. S.; Pun, S.; Stayton, P. S., Design and development of polymers for gene delivery. Nat. Rev. Drug Discov. 2005, 4, 581-593.

6. Städler, B.; Chandrawati, R.; Price, A. D.; Chong, S. F.; Breheney, K.; Postma, A.; Connal, L. A.; Zelikin, A. N.; Caruso, F., A Microreactor with Thousands of Subcompartments: Enzyme - Loaded Liposomes within Polymer Capsules. Angew. Chem. Int. Ed. 2009, 121, 4423-4426.

7. Trau, D.; Renneberg, R., Encapsulation of glucose oxidase microparticles within a nanoscale layer-by-layer film: immobilization and biosensor applications. Biosens. Bioelectron.2003, 18, 1491-1499. 
8. Johnston, A. P.; Cortez, C.; Angelatos, A. S.; Caruso, F., Layer-by-layer engineered capsules and their applications. Curr. Opin. Colloid Interface Sci.2006, 11, 203-209.

9. Nie, Z.; Petukhova, A.; Kumacheva, E., Properties and emerging applications of selfassembled structures made from inorganic nanoparticles. Nat. Nanotechnol. 2009, 5, 15-25.

10. Farokhzad, O. C.; Langer, R., Impact of Nanotechnology on Drug Delivery. ACS Nano 2009, 3, 16-20.

11. Shi, J.; Votruba, A. R.; Farokhzad, O. C.; Langer, R., Nanotechnology in Drug Delivery and Tissue Engineering: From Discovery to Applications. Nano Lett. 2010, 10, 3223-3230.

12. Chandrawati, R.; Caruso, F., Biomimetic liposome-and polymersome-based multicompartmentalized assemblies. Langmuir 2012, 28, 13798-13807.

13. Cui, J.; van Koeverden, M. P.; Müllner, M.; Kempe, K.; Caruso, F., Emerging methods for the fabrication of polymer capsules. Adv. Colloid Interface Sci. 2014, 207, 1431.

14. Björnmalm, M.; Cui, J.; Bertleff-Zieschang, N.; Song, D.; Faria, M.; Rahim, M. A.; Caruso, F., Nanoengineering Particles through Template Assembly. Chem. Mater. 2017, 29, 289-306.

15. Tan, S.; Cui, J.; Fu, Q.; Nam, E.; Ladewig, K.; Ren, J. M.; Wong, E. H.; Caruso, F.; Blencowe, A.; Qiao, G. G., Photocontrolled Cargo Release from Dual Cross-Linked Polymer Particles. ACS Appl. Mater. Interfaces 2016, 8, 6219-6228.

16. Wang, Y.; Angelatos, A. S.; Caruso, F., Template synthesis of nanostructured materials via layer-by-layer assembly. Chem. Mater. 2007, 20, 848-858.

17. Cui, L.; Wang, R.; Ji, X.; Hu, M.; Wang, B.; Liu, J., Template-assisted synthesis of biodegradable and pH-responsive polymer capsules via RAFT polymerization for controlled drug release. Mater. Chem. Phys. 2014, 148, 87-95. 
18. Tanner, P.; Baumann, P.; Enea, R.; Onaca, O.; Palivan, C.; Meier, W., Polymeric vesicles: from drug carriers to nanoreactors and artificial organelles. Acc. Chem. Res. 2011, 44, 1039-1049.

19. LoPresti, C.; Lomas, H.; Massignani, M.; Smart, T.; Battaglia, G., Polymersomes: nature inspired nanometer sized compartments. J. Mater. Chem. 2009, 19, 3576-3590.

20. Blanazs, A.; Armes, S. P.; Ryan, A. J., Self - assembled block copolymer aggregates: from micelles to vesicles and their biological applications. Macromol. Rapid Commun.2009, 30, 267-277.

21. Yu, B.; Wang, D. A.; Ye, Q.; Zhou, F.; Liu, W., Robust polydopamine nano/microcapsules and their loading and release behavior. Chem Commun. 2009, 44, 67896791.

22. Barbey, R.; Lavanant, L.; Paripovic, D.; Schüwer, N.; Sugnaux, C.; Tugulu, S.; Klok, H.-A., Polymer brushes via surface-initiated controlled radical polymerization: synthesis, characterization, properties, and applications. Chem. Rev. 2009, 109, 5437-5527.

23. Achilleos, D. S.; Hatton, T. A.; Vamvakaki, M., Light-regulated supramolecular engineering of polymeric nanocapsules. J. Am. Chem. Soc. 2012, 134, 5726-5729.

24. Richardson, J. J.; Björnmalm, M.; Caruso, F., Technology-driven layer-by-layer assembly of nanofilms. Science 2015, 348, aaa2491.

25. Zhang, X.; Chen, H.; Zhang, H., Layer-by-layer assembly: from conventional to unconventional methods. Chem. Commun. 2007, 14, 1395-1405.

26. Such, G. K.; Quinn, J. F.; Quinn, A.; Tjipto, E.; Caruso, F., Assembly of ultrathin polymer multilayer films by click chemistry. J. Am.Chem. Soc. 2006, 128, 9318-9319.

27. Kharlampieva, E.; Kozlovskaya, V.; Sukhishvili, S. A., Layer - by - Layer Hydrogen - Bonded Polymer Films: From Fundamentals to Applications. Adv.Mater. 2009, 21, 3053-3065. 
28. Kim, B.-S.; Park, S. W.; Hammond, P. T., Hydrogen-bonding layer-by-layerassembled biodegradable polymeric micelles as drug delivery vehicles from surfaces. ACS Nano 2008, 2, 386-392.

29. Such, G. K.; Johnston, A. P.; Caruso, F., Engineered hydrogen-bonded polymer multilayers: from assembly to biomedical applications. Chem. Soc. Rev. 2011, 40, 19-29.

30. Crespo-Biel, O.; Dordi, B.; Reinhoudt, D. N.; Huskens, J., Supramolecular layer-bylayer assembly: alternating adsorptions of guest-and host-functionalized molecules and particles using multivalent supramolecular interactions. J. Am. Chem. Soc. 2005, 127, 75947600.

31. Uhlenheuer, D. A.; Petkau, K.; Brunsveld, L., Combining supramolecular chemistry with biology. Chem. Soc. Rev. 2010, 39, 2817-2826.

32. Chen, G.; Jiang, M., Cyclodextrin-based inclusion complexation bridging supramolecular chemistry and macromolecular self-assembly. Chem. Soc. Rev. 2011, 40, 2254-2266.

33. Tan, S.; Ladewig, K.; Fu, Q.; Blencowe, A.; Qiao, G. G., Cyclodextrin - Based Supramolecular Assemblies and Hydrogels: Recent Advances and Future Perspectives. Macromol. Rapid Commun. 2014, 35, 1166-1184.

34. Harada, A.; Kamachi, M., Complex formation between poly (ethylene glycol) and $\alpha-$ cyclodextrin. Macromolecules 1990, 23, 2821-2823.

35. Harada, A.; Li, J.; Kamachi, M., Preparation and properties of inclusion complexes of polyethylene glycol with .alpha.-cyclodextrin. Macromolecules 1993, 26, 5698-5703.

36. Harada, A.; Takashima, Y.; Yamaguchi, H., Cyclodextrin-based supramolecular polymers. Chem. Soc. Rev. 2009, 38, 875-882.

37. Harada, A., Cyclodextrin-based molecular machines. Acc. Chem. Res. 2001, 34, 456464. 
38. Ooya, T.; Yui, N., Multivalent interactions between biotin-polyrotaxane conjugates and streptavidin as a model of new targeting for transporters. J. Control. Release 2002, 80, 219-228.

39. Araki, J.; Kataoka, T.; Ito, K., Preparation of a "sliding graft copolymer”, an organic solvent-soluble polyrotaxane containing mobile side chains, and its application for a crosslinked elastomeric supramolecular film. Soft Matter 2008, 4, 245-249.

40. Okumura, Y.; Ito, K., The polyrotaxane gel: A topological gel by figure - of - eight cross - links. Adv. Mater. 2001, 13, 485-487.

41. Tan, S.; Nam, E.; Cui, J.; Xu, C.; Fu, Q.; Ren, J. M.; Wong, E. H.; Ladewig, K.; Caruso, F.; Blencowe, A., Fabrication of ultra-thin polyrotaxane-based films via solid-state continuous assembly of polymers. Chem. Commun. 2015, 51, 2025-2028.

42. Dam, H. H.; Caruso, F., Construction and degradation of polyrotaxane multilayers. Adv. Mater. 2011, 23, 3026-3029.

43. Dam, H. H.; Caruso, F., Formation and degradation of layer-by-layer-assembled polyelectrolyte polyrotaxane capsules. Langmuir 2013, 29, 7203-7208.

44. Sabadini, E.; Cosgrove, T.; Taweepreda, W., Complexation between $\alpha$-cyclodextrin and poly (ethylene oxide) physically adsorbed on the surface of colloidal silica. Langmuir 2003, 19, 4812-4816.

45. Dreiss, C. A.; Cosgrove, T.; Newby, F. N.; Sabadini, E., Formation of a supramolecular gel between $\alpha$-cyclodextrin and free and adsorbed PEO on the surface of colloidal silica: Effect of temperature, solvent, and particle size. Langmuir 2004, 20, 91249129.

46. Takahashi, S.; Yamada, N. L.; Ito, K.; Yokoyama, H., Inclusion Complex of $\alpha-$ Cyclodextrin with Poly (ethylene glycol) Brush. Macromolecules 2016, 49, 6947-6952. 
47. Cui, J.; Björnmalm, M.; Liang, K.; Xu, C.; Best, J. P.; Zhang, X.; Caruso, F., Super -

Soft Hydrogel Particles with Tunable Elasticity in a Microfluidic Blood Capillary Model. Adv. Mater. 2014, 26, 7295-7299.

48. Pattanayek, S. K.; Juvekar, V. A., Prediction of adsorption of nonionic polymers from aqueous solutions to solid surfaces. Macromolecules 2002, 35, 9574-9585.

49. Lee, H.; Venable, R. M.; MacKerell Jr, A. D.; Pastor, R. W., Molecular Dynamics Studies of Polyethylene Oxide and Polyethylene Glycol: Hydrodynamic Radius and Shape Anisotropy. Biophys. J. 2008, 95, 1590-1599.

50. Becheri, A.; Lo Nostro, P.; Ninham, B. W.; Baglioni, P., The curious world of polypseudorotaxanes: cyclodextrins as probes of water structure. The Journal of Physical Chemistry B 2003, 107, 3979-3987.

51. Horský, J.; Porsch, B., Binding delocalization in polymer inclusion complexes of ring molecules: pseudopolyrotaxanes of $\alpha$-cyclodextrin and poly (ethylene glycol). J. Incl. Phenom. Macrocycl. Chem. 2005, 53, 97-102.

52. Yuen, F.; Tam, K. C., Cyclodextrin-assisted assembly of stimuli-responsive polymers in aqueous media. Soft Matter 2010, 6, 4613-4630.

53. Li, J.-H.; Wang, Y.-F.; Ha, W.; Liu, Y.; Ding, L.-S.; Li, B.-J.; Zhang, S., Cyclodextrin-based microcapsules as bioreactors for ATP biosynthesis. Biomacromolecules 2013, 14, 2984-2988.

54. Kobayashi, Y.; Katoono, R.; Yamaguchi, M.; Yui, N., Modulation of reversible selfassembling of dumbbell-shaped poly (ethylene glycol) s and $\beta$-cyclodextrins: precipitation and heat-induced supramolecular crosslinking. Polym. J. 2011, 43, 893-900.

55. McMullan, R. K.; Saenger, W.; Fayos, J.; Mootz, D., Topography of cyclodextrin inclusion complexes : Part I. Classification of crystallographic data of $\alpha$-cyclodextrin inclusion complexes. Carbohydr. Res. 1973, 31, 37-46. 
56. Tong, X.; Zhang, X.; Ye, L.; Zhang, A.-y.; Feng, Z.-g., Synthesis and characterization of block copolymers comprising a polyrotaxane middle block flanked by two brush-like PCL blocks. Soft Matter 2009, 5, 1848-1855.

57. Tardy, B. L.; Dam, H. H.; Kamphuis, M. M.; Richardson, J. J.; Caruso, F., SelfAssembled Stimuli-Responsive Polyrotaxane Core-Shell Particles. Biomacromolecules 2013, 15, 53-59.

58. Huh, K. M.; Cho, Y. W.; Chung, H.; Kwon, I. C.; Jeong, S. Y.; Ooya, T.; Lee, W. K.; Sasaki, S.; Yui, N., Supramolecular Hydrogel Formation Based on Inclusion Complexation Between Poly (ethylene glycol) - Modified Chitosan and a-Cyclodextrin. Macromol. Biosci. 2004, 4, 92-99.

59. Ping, Y.; Guo, J.; Ejima, H.; Chen, X.; Richardson, J. J.; Sun, H.; Caruso, F., pH Responsive Capsules Engineered from Metal - Phenolic Networks for Anticancer Drug Delivery. Small 2015, 11, 2032-2036.

TOC

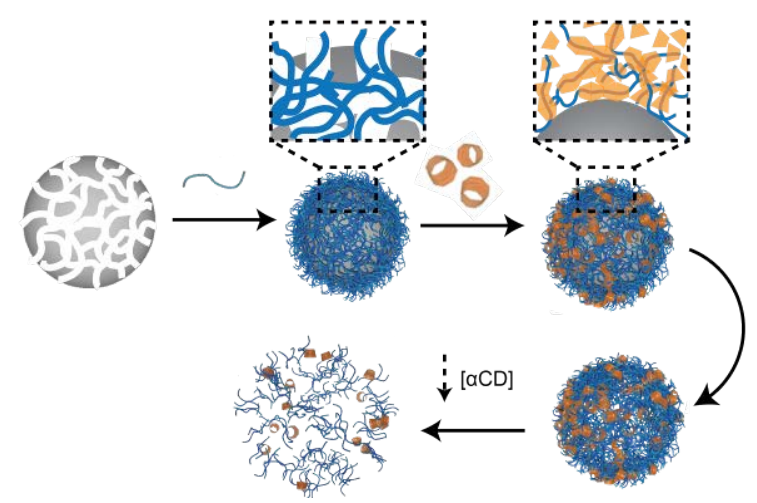

A method to assemble poly(ethylene glycol) (PEG)-based polyrotaxane (PRX) particles and capsules is reported. This is achieved by pre-absorbing PEG chains onto the template surface. 
Subsequently, $\alpha \mathrm{CDs}$ are threaded onto the PEG chains, allowing the formation of pseudopolyrotaxane particles stabilized by $\alpha \mathrm{CD}$ crystalline domains. The particle degradation rate, stability and cargo release can be tuned by simply varying the PEG $M_{\mathrm{w}}$ and endfunctionality or by the addition of small molecule cross-linkers. 